\title{
Cone mitochondria act as microlenses to enhance light delivery and confer Stiles-Crawford-like direction sensitivity
}

John M. Ball*, Shan Chen, Wei Li*

Retinal Neurophysiology Section, National Eye Institute, Bethesda, MD

*Corresponding authors

\begin{abstract}
Sensory systems that efficiently transduce physical energy into neural signaling are advantageous for survival. The vertebrate retina poses a challenge to such efficiency, featuring an inverted structure with multiple neural layers through which photons must pass, risking premature absorption or scattering. Moreover, mammalian photoceptors aggregate an unusual amount of mitochondria in the ellipsoid region immediately before the light-sensitive outer segments (OS). While these mitochondria are required to support the high metabolic demands of phototransduction, it is yet unknown their impact on light transmission. Here we demonstrate via direct live-imaging and computational modeling that such tightly packed mitochondria concentrate light to enter the OS for detection. Intriguingly, this "microlens"-like feature of cone mitochondria delivers light with an angular dependence akin to the Stiles-Crawford effect, an essential visual phenomenon that improves resolution. We thus establish an unconventional optical function for cone mitochondria, energy-producing organelles, providing insight into their role in the interpretation of noninvasive optical tools for vision research and ophthalmology clinics.
\end{abstract}

\section{Main Text}

The structure of the eye has long been central to debates concerning the nature and theories of evolution. One frequently discussed feature is the inverted structure of the vertebrate retina, in which light must pass through multiple neural layers before reaching the light-sensitive outer segment (OS) of the photoreceptor. Thus, there is a substantial evolutionary pressure for efficient light passage through the retina. Photoreceptor optics is a long-standing topic that predates our understanding of photoreceptors as the light-sensing neurons of the retina ${ }^{1}$; in the modern day, their waveguiding properties are instrumental to adaptive optics imaging, enabling the live resolution of individual human photoreceptors ${ }^{2,3}$. Thus, a central question is how retinal structures facilitate light delivery to 
the OS for detection. In rod nuclei, the compact arrangement of chromatin in nocturnal mammals is believed to provide an advantage for photon capture ${ }^{4}$, and Müller glia, which axially span the retina from its inner surface to the base of photoreceptor inner segments, have been shown to possess optical fiber-like light guidance properties ${ }^{5-7}$. Beyond these structures lie photoreceptor inner segments (IS), which comprise the last structure light must pass through before reaching the OS, and whose optical properties are believed to underlie their waveguiding capability ${ }^{8,9}$. In the outermost extent of the cone IS, many birds and reptiles (as well as some marsupials) possess large (2-5 $\mu \mathrm{m})$ oil droplets immediately proximal to $\mathrm{OS}^{10}$. Owing to their high index of refraction, oil droplets have long been suspected to serve as microlenses that concentrate light for detection in the $\mathrm{OS}^{11-14}$. However, modeling has more recently shown that this likely only increases light availability for nonpigmented oil droplets $^{15,16}$, whereas pigmented oil droplets sacrifice a significant amount of light intensity to provide spectral filtering of the light reaching the $\mathrm{OS}^{15,16}$. Despite these theoretical insights, direct experimental demonstration is rare.

Placental mammals lack oil droplets, instead possessing an abundance of mitochondria at this location, tightly packed in into an elongated bundle $(\mathrm{MtB})^{17,18}$. Given that mitochondria in mammalian cells ordinarily form a reticular network surrounding the nucleus ${ }^{19}$, such a high density of mitochondria at an unusual location is a peculiar feature of the retina. As with oil droplets, due to the high refractive index of lipid membrane 20,21 , these mitochondria have been speculated to likewise play an optical role $17,22,23$; however, unlike oil droplets, which comprise a uniform lipid syncytium, MtB contain numerous individual mitochondria with complex internal geometry, providing many refractive interfaces that may instead scatter light and compromise light delivery to OS. To date, due to the lack of a practical experimental preparation, a conclusive demonstration of their optical properties has yet to be presented. This question is highly relevant for human vision in which, despite the specialization of the fovea that displaces scattering elements from the light path, cone mitochondria remain an obstacle for light reaching the $\mathrm{OS}^{24}$.

\section{Light focusing by live cone mitochondria}

To investigate the optics of the cone MtB, we took advantage of a unique species-the thirteen-lined ground squirrel (GS)-for its cone-dominant (85\%) retina, featuring a single layer of photoreceptor cell bodies in its dorsal extent (Fig. 1A). Importantly, as a hibernator that endures near-freezing body 
temperatures for several months during the winter, the GS also features remarkable cold-tolerant cellular specializations that render ex vivo tissue stable for up to several days ${ }^{25-27}$. These properties allowed us to develop a preparation in which the optical properties of live, mitochondria-packed cone ellipsoids could be observed. This preparation involved the isolation of the cone IS layer by horizontal vibratome-sectioning of agarose-embedded GS retinal explants (Fig. 1B). The resulting samples featured patches of photoreceptors that often retained their native pseudo-hexagonal packing; their survival was verified by live labeling with tetramethylrhodamine ethyl ester (TMRE), which localizes to mitochondria due to their elevated inner membrane potential, providing both a clear indication of mitochondrial viability and a convenient marker for localization (Fig. 1B,D). Unexpectedly, such cones frequently lacked intact cell bodies, as assessed by post-hoc immunolabeling including DAPI (Suppl. Fig. 1) as well as during live imaging by Hoechst staining of cell nuclei (not shown) and the use of CellTracker, a fluorescent dye that permeates cell membranes and is subsequently converted to a cell-impermeant form (Fig. 1C). We speculate that the physical stress of sectioning may have caused photoreceptors to preferentially break at the cell body-IS junction (which is the narrowest part of the cell), after which the plasma membrane sealed to form an 'ellipsoidsome' containing live mitochondria (Fig. 1C cartoon; Suppl. Fig. 1A). This notion is supported by observations that this region is fragile and may be the source of detachment in certain retinal diseases ${ }^{28}$. Further, cone OS were typically detached by the removal of the RPE, likely due to the tight ensheathment of OS by RPE interdigitation between cones, and their presence vs. absence did not impart any evident systematic differences upon the optical data presented below. Thus, although light absorption will certainly benefit further from the waveguiding properties of the OS itself, here we focus on the optical role of the cone ellipsoid in the delivery of light to the OS.

By replacing the condenser of a confocal microscope with a dichroic mirror to direct collimated light from a blue-filtered white LED (490nm cutoff) toward the sample (Fig. 1B), we were able to acquire 3D image stacks of TMRE fluorescence within MtB as well as a 3D snapshot of the distribution of light energy that traverses them (Fig. 1C-D). Strikingly, we observed that LED light passing through cones was concentrated into beams of light many times brighter than the background intensity (Fig. 1D). Near the ellipsoid base, weak "halos" of light were observed that inverted near the MtB midpoint and ultimately coalesced to bright spots (termed "focal points" here; Fig. 1D). 

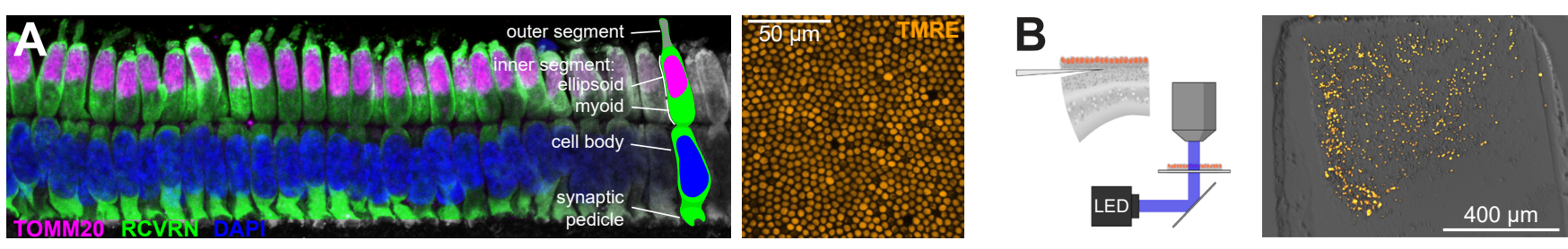

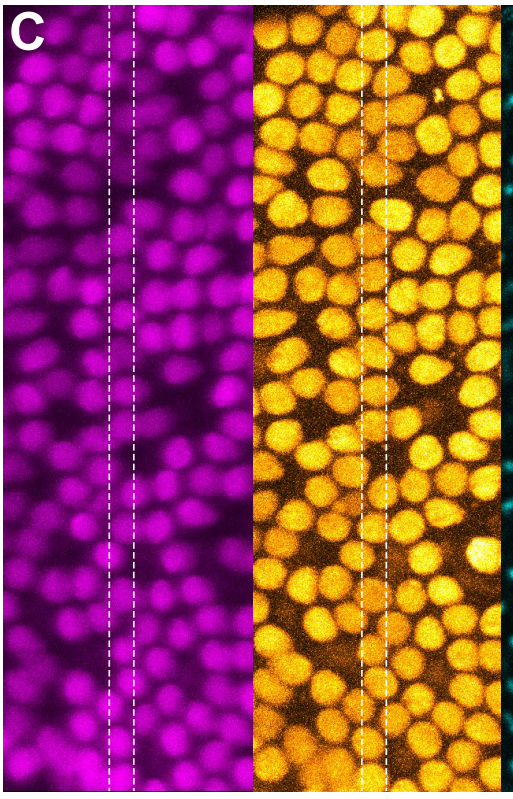

CellTracker

MitoTracker

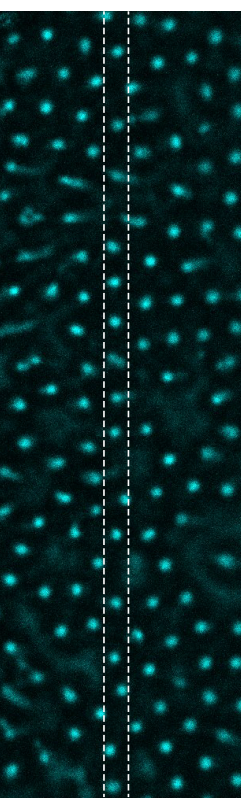

Light from LED

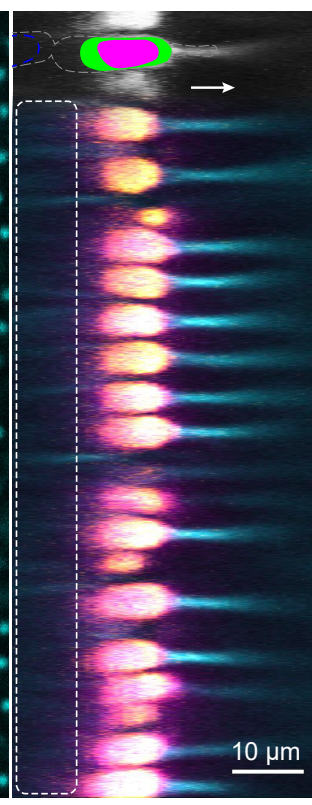

Ortho. Projection, Merge

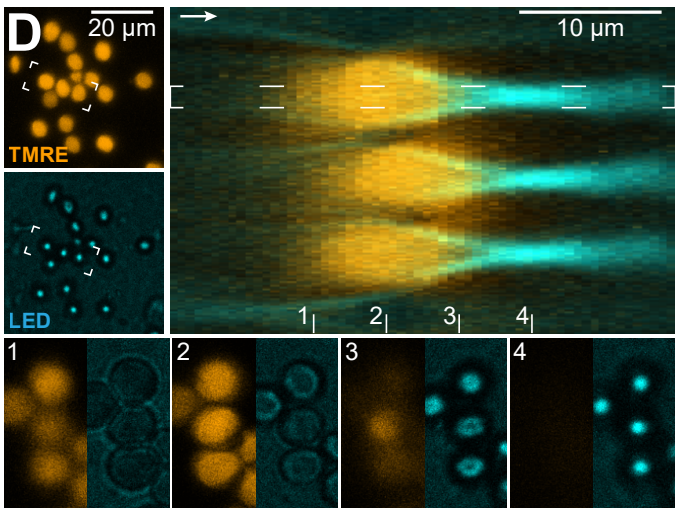

outer segment region (OSR)

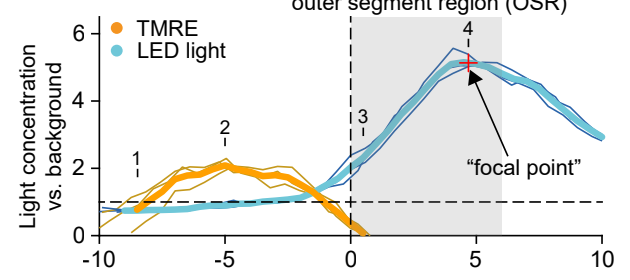

Depth relative to putative IS-OS border $(\mu \mathrm{m})$

\section{Figure 1: Light concentration by GS cone mitochondria.}

A) Cone mitochondria in dorsal GS retina. Left: Immunolabeled vertical section highlighting cone anatomy. Right: Live flat-mount view of TMRE-labeled mitochondria in photoreceptors. B) Horizontal sectioning and imaging of light transmission through agarose-embedded retinas. C) Concentration of light by photoreceptors in retinas sectioned as in B. MitoTracker localizes to mitochondria; CellTracker labels cytoplasm. In the orthogonal projection, note the absence of CellTracker labeling where the cone myoid region would ordinarily be expected (dashed line; compare to A). Arrow indicates the direction of light transmission. D) Quantification of light concentration factors in 3 exemplary cones. Flat-mount TMRE view is a maximum projection; LED image is a single plane at the peak concentration intensity. Orthogonal projection comes from the bracketed cones. Panels 1-4 depict features of light concentration at the depths indicated. Graph shows the relative light intensity (concentration factor) for the 3 cones (individual and average) as a function of depth from the distal IS tip in the $1.5 \mu \mathrm{m}$ diameter OS region (top cone, dashed line; see Methods). TMRE signal is arbitrarily scaled for comparison. 
These focal points were located a few $\mathrm{mm}$ beyond the distal end of the IS and had a half-width of $\sim 1$ $\mu \mathrm{m}$ (Fig. 1D) - very similar to the size of the GS cone OS ( $\sim \mu \mathrm{m}$ long and $1.5 \mathrm{~mm}$ in diameter; see Fig. 2A). Such placement of a concentrated beam of light would greatly enhance entry of photons into the OS and thus, their detection. In fact, cones with shorter focal lengths would be better suited to couple this intense beam of light into the proximal end of the OS, which may then guide this light along its length, maximizing detection. Notably, in rare samples, sectioning yielded patches of isolated cones with intact cell bodies; cones in these patches featured short focal lengths (Suppl. Fig. 2), supporting the prediction that cones concentrate light at short distances in vivo.

\section{Optical consequences of mitochondrial remodeling}

To further probe the relationship between MtB structure and this concentration of light, we took advantage of the hibernating GS, whose cone mitochondria have been reported to undergo structural changes that include a reduction in total number and/or volume ${ }^{29-31}$. Such structural remodeling of the MtB may lead to measurable optical consequences. To obtain high quality 3D reconstructions of mitochondria, which yielded structures with a resolution suitable for computer simulation of light transmission (see details below), we applied serial block-face electron microscopy (SBEM) to dorsal cones in both an active and a hibernating GS (Fig. 2A-B). Mitochondria in hibernating GS cones were as numerous as those in awake GS but were individually smaller, resulting in a lower total MtB volume in hibernating cones (30\% lower; see Fig. 2B-C). Importantly, mitochondria morphology in hibernating GS cones was qualitatively different; whereas mitochondria in active GS appeared elongated and wellorganized, those in hibernating GS appeared distorted and markedly less well aligned (Fig. 2B). Detailed analysis indicated exceptional alignment among active GS cone mitochondria; approximately $75 \%$ of mitochondria skeleton "branches" reconstructed from awake cones deviated by less than $15^{\circ}$ from overall MtB orientation (see Methods, Suppl. Fig. 3 for details), compared to only 30\% in hibernating cones, indicating considerable disorganization (Fig. 2D; Suppl. Fig. 4).

Concomitantly, we also observed optical differences between awake and hibernating GS cones. MtB from hibernating GS cones concentrated light less effectively than did those from active GS (Fig. 2E). While focal lengths were wide-ranging but similar in both awake and hibernating samples (active $5.8 \pm$ $2.2 \mu \mathrm{m}$; hibernating $6.0 \pm 2.5 \mu \mathrm{m})$, peak intensity was higher in active ( $6.6 \pm 0.8$-fold brighter than background) than in hibernating samples (5.2 \pm 0.5 -fold). 


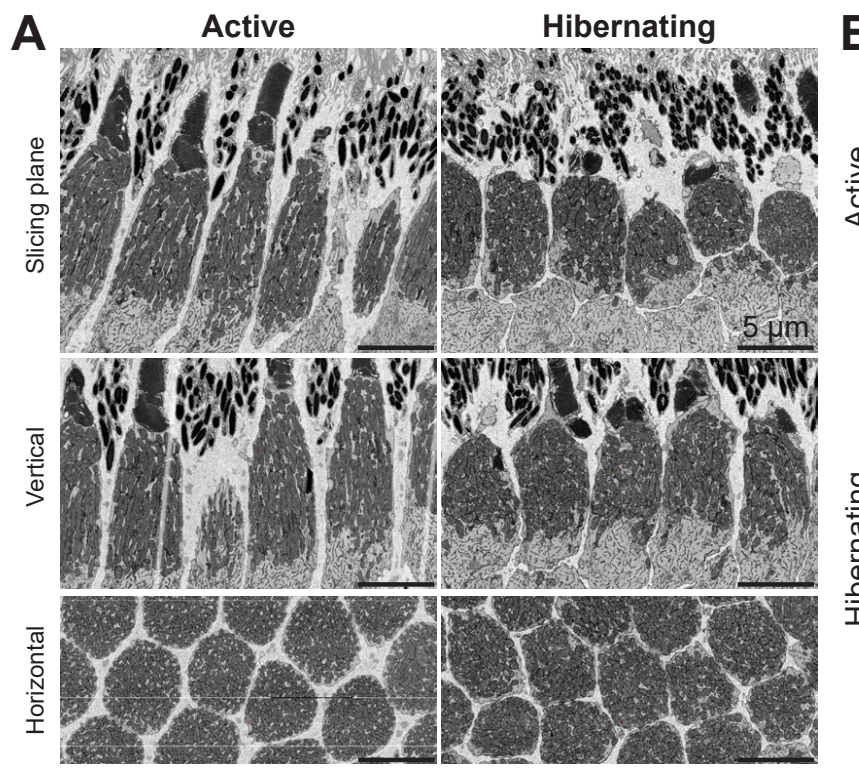

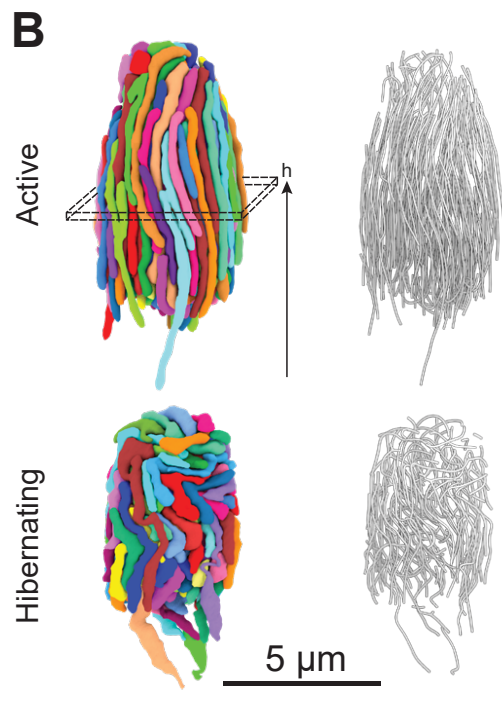

Reconstruction Skeleton
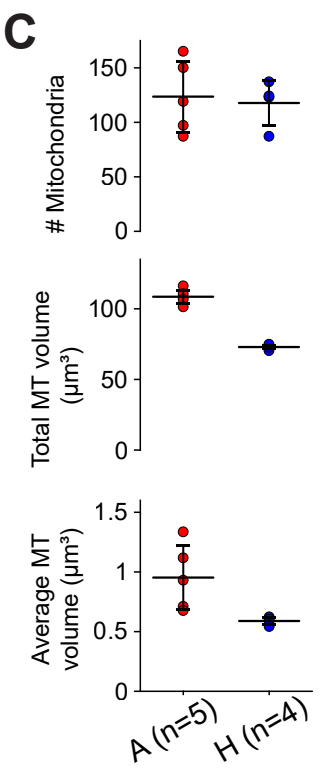

D MT skeleton
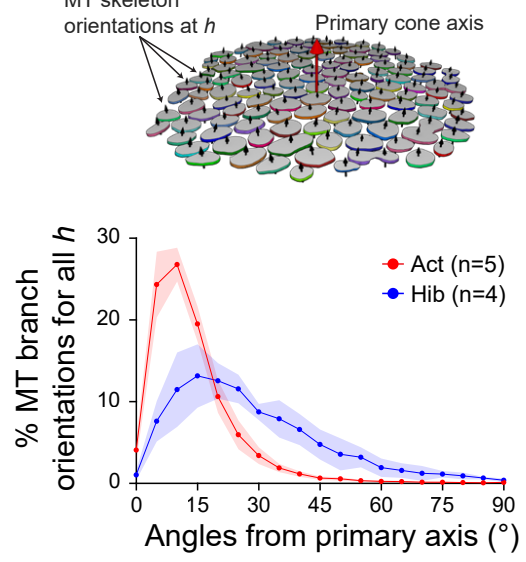

E Experimental light concentration by active vs. hibernating GS cones
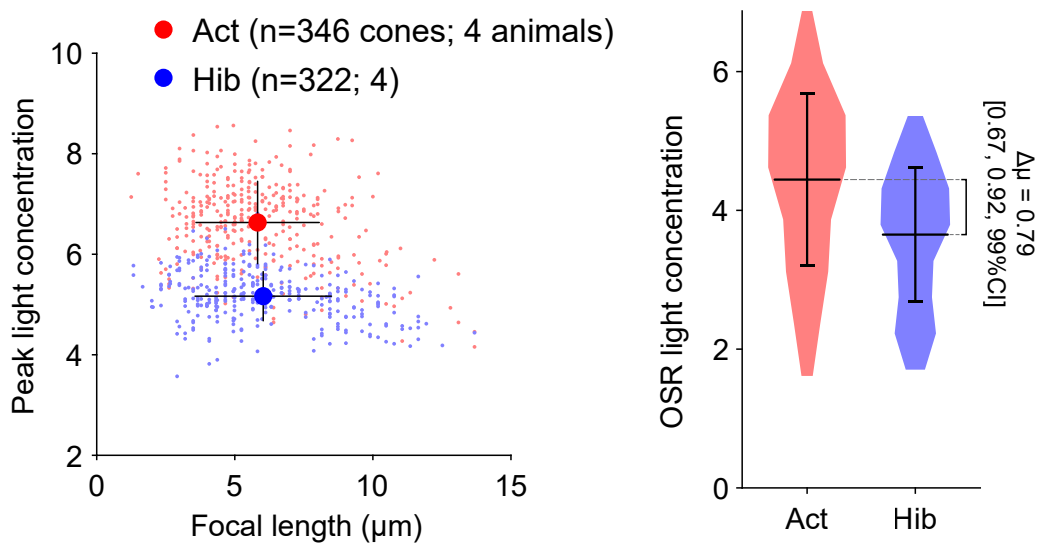

Figure 2: Cone mitochondrial structural changes correlate with hibernation-induced decreases in light concentration.

A) SBEM images of GS cone mitochondria. Vertical and horizontal images are software projections. B) Example reconstructions (segmented 3D models and skeletonizations) of all mitochondria from sample cones. C) Morphological quantification of reconstructed mitochondria. Data shown as mean \pm standard deviation. Statistical comparison not performed here (see Methods). D) Quantification of mitochondrial alignment in cones using skeletonizations. Diagram depicts the scheme for measuring mitochondria branch orientation at an example cone height $h$ (see panel B). Graph shows histograms (mean \pm standard deviation; shaded regions) of mitochondria branch angles throughout reconstructed cones. E) Experimental light concentration comparison for active vs. hibernating GS cones (see Fig. 1). Scatterplot shows cone focal points and mean \pm standard deviation for each condition. Violin plot shows the distributions of average light concentration in the OSR (see Methods for an explanation of statistics). 
In order to estimate the overall effect of these differences on the light theoretically available to a cone OS, we integrated the total light within a cylinder approximating the anatomical outer segment region (OSR; with dimensions as described above). Average light concentration in the OSR was 22\% higher in active samples ( $4.44 \pm 1.25$ vs. $3.65 \pm 1.0$-fold-increase). This difference supports the hypothesis that the elongated, parallel organization of mitochondria in cone MtB appears to enhance the concentration of light for detection in the OS.

In such experiments, differences in MtB optics between active and hibernating GS may result from a confounding mixture of differences in gross morphology as well protein concentration ${ }^{32,33}$ or even altered cristae structure ${ }^{34}$. To assess specifically the impact of MtB morphology on light concentration, we turned to computational methods. To this end, we translated reconstructed MtB models into 3D fine-grained dielectric structures (Fig. 3A, Suppl. Fig. 3) suitable for use with code libraries from MEEP (MIT Electromagnetic Equation Propagation FDTD simulator), which divides time and space into a grid of points within which Maxwell's equations of electromagnetism are calculated ${ }^{35}$. The electromagnetic energy distribution in this grid, reached upon the introduction of a $450 \mathrm{~nm}$ continuous-wave energy source at the proximal end of the cone and allowing the resulting propagating waves to settle, provides a theoretical, 3D view of the passage of blue light through these structures that could be quantified with the same techniques used for our confocal imaging data (Figs. 1-2).

Light concentration by cones in simulations matched what we observed experimentally (Fig. 3B). Importantly, simulations permitted us to assess specifically the relative contribution of MtB structure and cone IS to the light concentration capability of cones. Cone IS without mitochondria were nonetheless capable of gathering light; however, in these simulations, concentration occurred at considerably longer focal lengths where the majority of the electromagnetic energy would fail to enter and be guided by or absorbed in the OS (Fig. 3B-C). In contrast, simulations of isolated MtB (as if the cone IS membrane had been dissolved, leaving behind only structurally intact mitochondria) exhibited light concentration much more similar to that of intact cones (Fig. 3C), suggesting that MtB play a major role in light concentration for entering OS.

Corroborating our experimental imaging results, simulations from hibernating GS cones likewise demonstrated weaker light concentration in the OSR (5.8 \pm 0.6 -fold vs. $7.8 \pm 1.2$-fold for active GS 
cones; Fig. 3D). This difference resulted both from lower peaks and longer focal distances - slightly differing from our experimental results, which largely reflect a reduction in peak intensity (Fig. 2E). This is likely due to other changes accompanying the structural alterations in mitochondria of hibernating GS cones, such as protein concentration ${ }^{36}$ or inner matrix volume ${ }^{34}$, which will affect mitochondrial refractive index, in turn altering focal lengths and peak intensity as demonstrated by simulations (Suppl. Fig. 5).

More importantly, isolated MtB from active GS cones were potent light gatherers, retaining $80 \%$ of the OSR light concentration factor seen in intact cone simulations (Fig. 3E); in contrast, isolated MtB from hibernating GS were only half as effective. Emphasizing this differential effect of MtB structure upon optics, cone IS devoid of mitochondria were equally ineffective at concentrating light, regardless of whether they came from active or hibernating GS (Fig. 3E). These results indicate that MtB with highly ordered spatial organization can significantly improve its ability to concentrate light. Indeed, simulations indicated that such organization was superior for light concentration compared to volumematched globular mitochondria (Suppl. Fig. 6). Interestingly, a single "megamitochondrion"

(Suppl. Fig. 6), an exaggerated configuration resembling the giant mitochondria seen in some cones of the tree shrew ${ }^{37}$ and zebrafish ${ }^{38,39}$, similar also to the "ellipsosomes" present in other species ${ }^{40}$, proved to be a highly effective configuration for light concentration.

\section{Directionality of mitochondrial focusing and the Stiles-Crawford effect}

Taken together, MtB configurations that favor tight packing and elongation appear to maximize the potential for photon delivery to the OS, likely by reducing the number of refractive interfaces they encounter. This strategy approximates that employed by species bearing oil droplets, which act as microlenses with a homogeneous elevated refractive index ${ }^{10}$. Emphasizing this microlens-like property of the MtB, we observed-as has been demonstrated for oil droplets ${ }^{11}$ - that cone ellipsoids reproduced and inverted an image plane (generated by placing a mask with a slit in front of the LED) near their focal point (Fig. 4A). This observation likewise suggests that as with a lens, light incident upon MtB at an angle that deviates slightly from its optical axis may be nevertheless focused along that misaligned axis. In well-preserved retinal sections, alignment of inner and outer segments is typical (Fig. 4B, see also references 41-43), and recently it has been demonstrated that a light-dependent developmental process ensures such alignment ${ }^{44}$. 
A

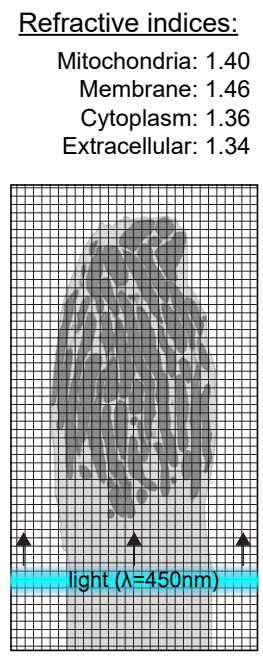

3D grid voxel size: $20^{3} \mathrm{~nm}^{3}$
B

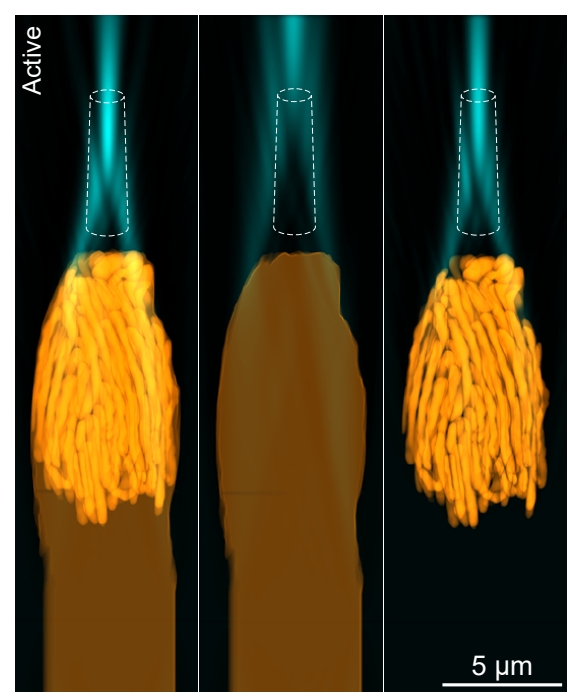

C
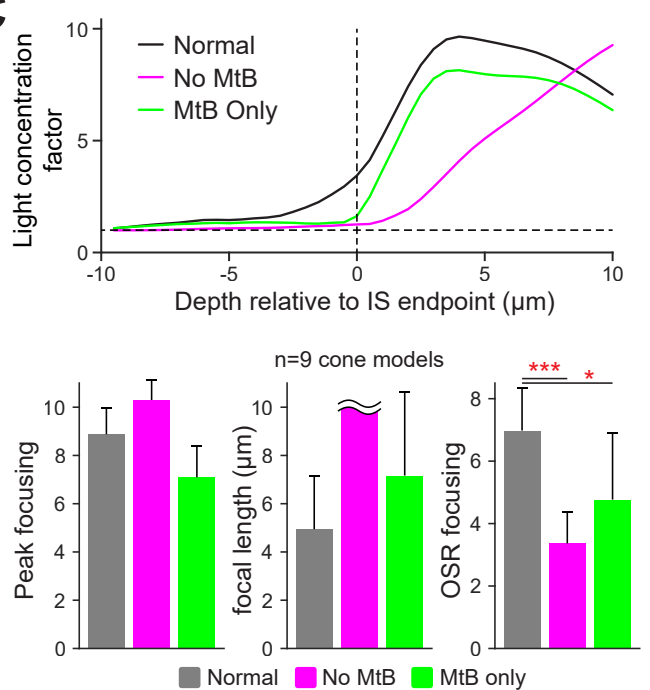

E
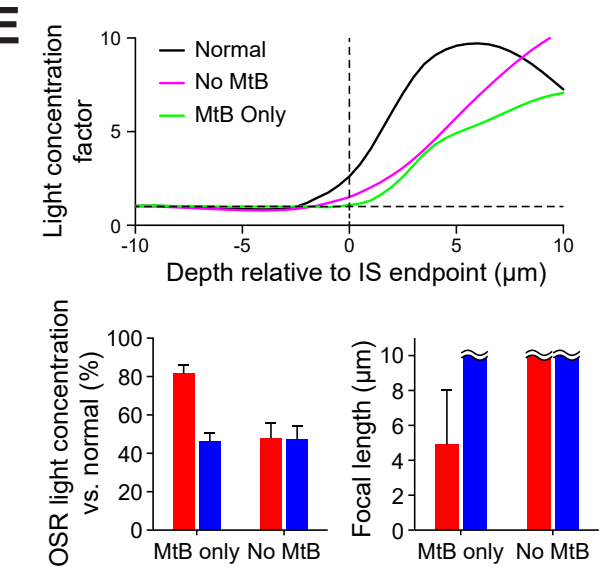

Figure 3: Electromagnetic simulations of light concentration by GS cone mitochondria.

A) Simplified 2D schematic of the 3D FDTD simulation framework. B) Example light gathering by a reconstructed cone from active GS; from left-to-right: Intact, without mitochondria, suspended mitochondria only. Dashed shape depicts approximate OS region. C) Quantification of simulated light gathering. Top graph depicts light concentration factor profiles for simulations in B. Bar graphs show aggregate measures across all reconstructions $(n=9)$. Values are mean \pm standard deviation; wavy lines indicate apparent focal lengths that fell beyond the upper boundary of the simulation volume $(>10$ $\mu \mathrm{m})$. D) Simulated light gathering by an example cone from hibernating GS. Note the considerable decrease in intensity without the cell membrane (see also panel E). Graphs depict light gathering comparisons between active and hibernating GS cones (mean \pm standard deviation). E) Mitochondriadependence of simulated light gathering. Top profile plots are for simulations in D (left). Bottom graphs show change in concentration factor for active vs. hibernating GS cones with either the MtB or the IS cell membrane removed. 
Therefore, light arriving along this axis will be maximally concentrated onto the OS; however, light entering at an angle will partially miss the OS (Fig. 4B). This observation could potentially contribute to the Stiles-Crawford effect (SCE), an psychophysical phenomenon rooted in photoreceptor optics that has captivated vision scientists for nearly 90 years ${ }^{8,45}$.The SCE, which dictates that photoreceptors (especially cones) are less sensitive to rays of light entering the pupil peripherally, is believed to improve visual resolution and reduce veiling background ${ }^{8}$ (Fig. 4C). Such directional sensitivity of light perception is canonically attributed to the waveguiding properties of cones ${ }^{45}$. However, while the relatively high refractive index of mitochondria has been noted ${ }^{17,46-48}$, their role in SCE remains speculative, ranging from confounding the SCE via scattering ${ }^{9,46}$ to instead enhancing $i^{17}$. Here we hypothesize that coaxial light concentration by the MtB could produce SCE-like direction sensitivity, and the amount of light rendered unavailable to the OS due to misalignment would be affected by the extent of MtB focusing (Fig. 4B).

To test this, we simulated light transmission through GS cone models tilted by angles up to $10^{\circ}$ and measured the energy density of light concentrated along the incident axis as well as in the apparent region in which the OS would reside if it were aligned with the tilting of the IS (Fig. 4D-E). We calculated the direction sensitivity parameter $\rho$, which originates from a fit to psychophysical SCE direction sensitivity data and describes the steepness of the SCE curve, and which ranges $\sim 0.05-0.09$ $\mathrm{mm}^{-2}$ for human retina (Fig. 4C, see also references 8,49-50), while smaller values can indicate pathological disruption of photoreceptors ${ }^{51}$. Indeed, tilting had little effect on the amplitude of light concentration; however, the loss of light available to the shifted OS region as a function of tilting angle agreed well with the SCE, yielding $\rho=0.052$ across all cone models (Fig. 4E). With the longer "focal lengths" produced in the absence of the MtB (see Fig. 3C), the loss of light at the OSR due to tilting was diminished, effectively “impairing" direction sensitivity ( $\rho=0.028$; Fig. 4F).

We further corroborated these theoretical results experimentally. Due to preparation and handling in live-imaging experiments, photoreceptors displayed apparent MtB tilts of up to $15^{\circ}$ (Fig. $4 \mathrm{G}$ ). While concentrated light beams in these cells appeared only minimally disturbed by misalignment, the light intensity within the presumptive OS showed a similar direction sensitivity (Fig.4H). 

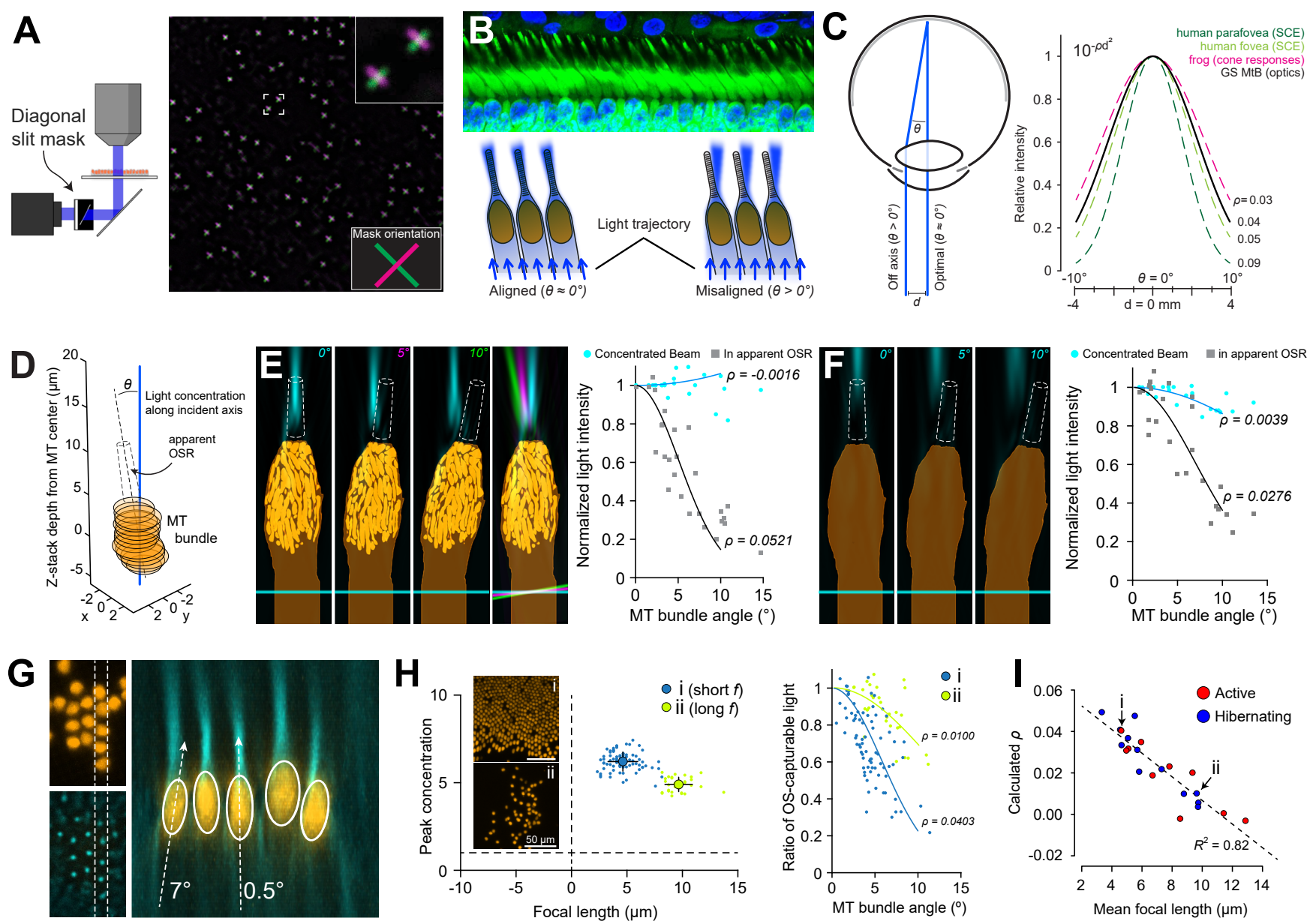

Figure 4: Directionality of light focusing by GS cone mitochondria.

A) Reproduction of an inverted image plane (slit mask) at the focal points of cone MtB. Image is the overlaid result of two separate acquisitions with the mask reversed before the second acquisition. B) Conceptual illustration of angle-dependent concentration of light upon the cone OS. C) Diagram of the Stiles-Crawford effect and directionality. Angular dependence of light focusing shown here in GS is overlaid upon directionality tuning curves based on measurements in human SCE ${ }^{47,51,52}$ and frog cone light responses ${ }^{17}$. D) Schematic of the quantification of direction sensitivity for cone light concentration in 3D image stacks (see Methods). E) Direction sensitivity of OSR light intensity in simulated GS cones. Left panels show example simulations with models tilted by the indicated angles. Overlay shows the simulations rotated in register with one another in different colors for comparison. Graph shows the direction sensitivity fit analysis (compare to panel C) across simulations for all cone models ( 3 angles per cone model). F) Direction sensitivity of simulated cone light concentration without mitochondria. G) Concentration by cone mitochondria along the axis of light incidence. Annotations are simplified angle measurements in the 2D plane used here only for illustration. $\mathbf{H}$ ) Increased direction sensitivity of OSR light concentration by cones in a sample with short average focal lengths ( $i$; short $f$ ) compared to one with long average focal lengths (ii; long $f$ ). I) A strong correlation between mean focal length and apparent direction sensitivity (calculated $\rho$ ) across GS samples. 
Moreover, as with simulations, samples with shorter focal lengths (likely reflecting healthier, more intact conditions) yielded larger $\rho$ values, which were close to our simulation value and within the range of those reported for human SCE (Fig. 4H). This relationship between focal length and $\rho$ was linear and strongly correlated across both active and hibernating TLGS ( $R^{2}=0.82$, Fig. 4I). Interestingly, single cone light responses in the frog retina have been demonstrated to demonstrate such directional sensitivity (Fig. 4C) ${ }^{13}$, highlighting the capability of short, blunter cones to possess directional tuning. It is tempting to speculate that focusing by oil droplets may likewise be responsible for such directiondependent light delivery to the OS; however, simulations concerning the oil droplet-bearing cones in chicken exhibit a much broader directional tuning curve $\left(>85 \% \text { light energy at up to } 15^{\circ}\right)^{15}$. Thus, this question requires further study with respect to the optics of oil droplets.

This demonstration of a role for MtB in the SCE-like angular dependence of concentrated light delivery to the cone OS provides necessary conceptual refinement to the waveguiding properties of cones. Central foveal (foveolar) cones are believed to possess more sparsely packed mitochondria compared to those even immediately outside the most central fovea ${ }^{17,54,55}$. Interestingly, SCE is also weaker for foveolar cones than those outside the central fovea (Fig. 4C) 52,53,56,57, which are shorter, have larger diameters, and feature prominent GS cone-like mitochondria bundles ${ }^{8,17,55}$. Foveolar cones may however benefit from their long and narrow IS, as our simulations indicate that simply lengthening the inner segment of the GS cone shortens its effective focal length (Suppl. Fig. 7). Nonetheless, GS-like cone morphology is common among vertebrates, appearing even in lampreys ${ }^{58}$. Taken together, direction sensitivity in non-foveolar cones-as well as in non-primate species-may require the MtBbased optical phenomenon demonstrated here.

\section{Conclusion}

In sum, through live-imaging and simulation, we demonstrated that spatial organization of cone mitochondria allows the MtB to efficiently concentrate light to enhance IS-OS coupling. Such a role constitutes a fascinating phenomenon whereby the mitochondria of mammalian cones fulfill a dual purpose: their well-established metabolic role as well as an unconventional optical effect. This clear optical role for cone mitochondria may shed light upon the interpretation of their relevance in ophthalmological diagnostic tools ${ }^{18,59}$. The lens-like property demonstrated here for GS cone 
mitochondria also exhibits a SCE-like angular dependence of light delivery to the OS. This sharpening of directional tuning by the MtB, especially given the strong correlations shown here between directionality and focal length (Figure 4I) and between focusing and MtB refractive index (Figure S5), lends support to the use of SCE as a non-invasive measurement for the diagnosis of retinal degenerations, many of which entail dysfunctions that may impart either structural or biochemical changes to mitochondria, both of which will have optical consequences ${ }^{51,60}$.

\section{Acknowledgments}

This work was funded by the intramural research program of the NIH. We thank Gerald Westheimer and Johnny Tam for helpful discussions and critical review of this manuscript. The simulation work for this study utilized the computational resources of the NIH HPC Biowulf cluster (https://hpc.nih.gov); we would like to extend special thanks to Susan Chacko for considerable assistance configuring and maintaining the MEEP simulation code libraries that made this study possible. We would like to thank the diligent work of the NIH animal facility staff for year-round care of the ground squirrel colony and hibernaculum. Mitochondria reconstructions were performed by the persistent, careful work of Anurag Goel, Jennifer Du, and David Lu under the supervision of Talia Kaden. 


\section{Methods}

$\underline{\text { Animal care and use }}$

Female thirteen-lined ground squirrels (Ictidomys tridecemlineatus), ranging 7-15 months old (100-300 g) at the time experiments were conducted were used for this study. Females were used due to their relative abundance in these litters, which were purchased from a colony managed at the University of Wisconsin-Oshkosh animal facility $\underline{61}$.

All animal procedures were pre-approved by the Institutional Animal Care and Use Committee of the National Eye Institute (NEI) and conformed to the provisions of the Animal Welfare Act (NIH/DHHS). From May to August, squirrels were housed individually on Aspen Sani-Chips bedding (depth 1") in a holding room set to $72^{\circ} \pm 4^{\circ} \mathrm{F}\left(22^{\circ} \mathrm{C}\right)$ and $30-70 \%$ humidity. Enrichment consisted of one handful of Enviro-dri bedding and a tunnel made of PVC pipe. Lighting was supplied by full spectrum bulbs to mimic natural daylight, with a photoperiod matching that prevailing in Oshkosh, Wisconsin. From March through August, squirrels were fed cat chow ad libitum. In early July, experimental animals were equipped with subdermal implants for identification and body temperature measurements (BMDS IPTT-300; Bio Medic Data Systems, Seaford, DE).

From September through February, the cat chow ration was regulated to $60 \mathrm{~g}$ per week, and their body temperatures periodically measured. When animals displayed early signs of hibernation/torpor (became sluggish or unresponsive), their cages were transferred to a dark hibernaculum set at $39^{\circ} \pm 3^{\circ}$ $\mathrm{F}\left(4-5^{\circ} \mathrm{C}\right)$ and $30-70 \%$ humidity and monitored daily for short periods under dim red light. Animals that remained torpid for 3 consecutive days were transferred, with their nest but without food or water, to a 4.4-quart Handi-Box that served as their nest chamber for the duration of their hibernation period.

Euthanasia was carried out on animals exposed to $6 \mathrm{~L} / \mathrm{min}$ of isoflurane in a $30-\mathrm{L}$ glass cage lined with wood shavings until deeply sedated, followed by decapitation using a guillotine.

\section{$\underline{\text { Retinal sample preparation }}$}

Following euthanasia, GS eyes were removed, and posterior segment eye cups were dissected out of enucleated eyes and placed in Hibernate A medium (BrainBits LLC, Springfield, IL). Retinas were dissected out of eye cups, and horizontal sections from dorsal retina that isolated photoreceptor layers were prepared as follows: First the dorsal region was cut away from the optic nerve head and the inferior retina, and the retinal pigment epithelium was gently removed, taking care to preserve the natural orientation of photoreceptors. The dorsal retina was then cut into small ( 1-2mm square) sections which were then laid flat (photoreceptor side-down) on a standard microscope slide and coated with low melting-point agarose (1\% w/v in HEPES-buffered Ames' medium; Sigma-Aldrich, St. Louis, MO) kept at $50^{\circ} \mathrm{C}$ using a water bath. This was done with the microscope slide placed on an ice pack to speed agarose hardening. Multiple such embedded samples were then placed on a prepared block of standard agarose and coated with more low-melting point agarose to fix them in place. This block was then immersed in the reservoir of a vibratome (Leica VT1000 vibratome; Leica Biosystems, Buffalo Grove, IL) containing room temperature Hibernate A. This block was iteratively sliced and 
inspected until only photoreceptors remained, at which point a final cut was made to free the photoreceptor-containing slice from the agarose block and invert it photoreceptor side-up for imaging.

Agarose-embedded samples were incubated for 30-60 minutes at $4^{\circ} \mathrm{C}$ with vital dyes diluted in Hibernate A prior to imaging. Dyes typically included tetramethyl rhodamine, ethyl ester (TMRE 50 mM; Thermofisher), Hoechst 33342 nuclear stain (3 mM, Thermofisher); alternatively MitoTracker Green ( $250 \mathrm{nM}$, Life Technologies) and CellTracker Orange (2 mM, Life Technologies) were used. Following this incubation, samples were gently rinsed with cold HEPES (10mM) -buffered Ames' solution (Sigma-Aldrich), placed into fresh Hibernate A with a low concentration of TMRE (5 mM) and returned to the refrigerator until imaging. Incubation at $35^{\circ} \mathrm{C}$ accelerated dye loading, but samples thrived better at lower temperatures and were thus kept in the refrigerator. For imaging, samples were transferred to dishes containing HEPES-buffered Ames' and 5 mM TMRE. Ames' medium was used instead of Hibernate A for imaging because the use of Phenol Red in the latter for $\mathrm{pH}$ monitoring may introduce undesired optical effects. For all data presented here, live images were acquired $<8 \mathrm{~h}$ following initial euthanasia.

\section{Confocal imaging of light transmission through live photoreceptors}

Agarose-embedded samples were placed photoreceptor side-up in glass-bottom dishes under an upright laser-scanning confocal microscope (LSM 510; Carl Zeiss Microscopy, White Plains, NY). In order to provide an external transmitted light source during imaging, the condenser was replaced with a custom housing containing a beam combiner to direct light from a collimated "cool white" LED (Mightex LCS-5500-12-22, Mightex Systems, Pleasanton, CA) from below the sample toward the microscope objective, mimicking the normal direction of light passage through the retina. Light from the LED was typically filtered to short (blue) wavelengths using a dichroic color filter (ThorLabs FD1B, 490nm cutoff; ThorLabs, Newton, NJ)-occasionally this filter was removed to simultaneously observe light concentration by cones across multiple wavelength bands. A glass diffuser was placed between the collimating lens and the blue filter to ensure uniform illumination; preliminary results without a diffuser resulted in the reproduction of the 4-emitter LED pattern at the focal length of mitochondria, leading to erroneous measurements of light concentration ability (see also Fig. 4A).

For TMRE-loaded samples, acquisition was performed on two acquisition channels in Zeiss Zen software, with the blue transmitted light filtered through a 435-485nm emission bandpass ("blue channel"), and TMRE excited using a 561nm laser and acquired using a 575-630nm bandpass ("TMRE channel"). Transmitted light from the LED was not visible on the TMRE channel or vice-versa. 3D volume stacks ("Z-stacks") were acquired from both channels using standard parameters for TMRE, and a pinhole size of $126 \mathrm{~mm}$ for the blue channel. Prior to each acquisition, the intensity of the transmitted LED was adjusted while focusing on the bright puncta of concentrated light above cones in order to prevent saturation of that channel. Z-stack acquisition times ranged from 10-30 min.

Samples including MitoTracker Green were also excited with the 488nm laser line and the green channel acquired behind a 500-550nm bandpass. Blue light from the LED was visible on the green channel, but was clearly distinguishable from MitoTracker labeling, which in turn was not visible in the transmitted light channel. For acquisitions of light concentration by cones in multiple wavelength 
bands, no laser excitation was used, but the blue LED filter was removed and transmitted light was simultaneously acquired on multiple channels filtered to $435-485 \mathrm{~nm}, 500-550 \mathrm{~nm}$, and $650-710 \mathrm{~nm}$. In order to spatially register these images with cones, the blue LED filter was then reinserted and the 435$485 \mathrm{~nm}$ channel re-acquired simultaneously with TMRE fluorescence. The two blue channels were then used to later register the two image stacks for analysis.

Agarose gel possesses a refractive index ranging from $1.335-1.340 \underline{62,63}$, similar to the value used in FDTD electromagnetic simulations for the extracellular space surrounding cones (see below).

\section{$\underline{\text { Immunohistochemistry }}$}

Following live imaging, samples were fixed in 4.0\% PFA for at least $1 \mathrm{~h}$ and then processed for postfixation imaging to verify cone structures. Samples were blocked with $4 \%$ normal donkey serum (Jackson ImmunoResearch Laboratories, West Grove, PA) in PBS containing 0.1\% Triton X-100 (Sigma) for $2 \mathrm{~h}$. Samples were incubated at least $4 \mathrm{~h}$ with antibodies against the mitochondrial membrane protein Tomm20 (ab186734 or ab56783; abcam, Cambridge, MA), as well as either cone arrestin (Arr3 antibody sc-54355; Santa Cruz Biotech, Dallas, TX) or recoverin (Rcvrn antibody AB5585; Millipore, Burlington, MA) to label cone photoreceptors. Samples were then incubated at least $4 \mathrm{~h}$ in fluorophoreconjugated secondary antibodies raised in donkey against rabbit, mouse, and/or goat primary antibodies (Jackson) before mounting. Brief incubation with DAPI (ThermoFisher) was included during final sample washes to label nuclei. Low-resolution images taken during live imaging were used to reidentify patches of photoreceptors and verify the absence or presence of cone cell bodies. Images were acquired using an inverted confocal microscope (LSM 780, Zeiss).

\section{Serial block-face scanning electron microscopy (SBEM)}

Dorsal retinal samples were excised as described above, fixed with $4 \%$ paraformaldehyde and $2.5 \%$ glutaraldehyde, and provided to Renovo Neural, Inc (Cleveland, $\mathrm{OH}$ ) for SBEM imaging $\underline{64}$. Samples were provided from one active and one hibernating GS as determined above; animals were euthanized in late March when both hibernating and non-hibernating GS were available. The block size and resolution of the resulting SBEM datasets were: Active, $28.7 \times 28.7 \times 15.0 \mathrm{~mm}, 7 \times 7 \times 50 \mathrm{~nm}$ resolution; Hibernating block \#1, $21.5 \times 28.7 \times 11.0 \mathrm{~mm}, 7 \times 7 \times 30 \mathrm{~nm}$ resolution; Hibernating block \#2, $21.5 \times 28.7$ $\times 10.0 \mathrm{~mm}, 7 \times 7 \times 30 \mathrm{~nm}$ resolution.

\section{Cone mitochondria reconstructions and analysis}

Images acquired with SBEM were loaded into Reconstruct v1.1

(https://synapseweb.clm.utexas.edu/software-0) 65 and/or IMOD v4.9.0

(https://bio3d.colorado.edu/imod/) ${ }^{66}$ for manual segmentation. Approximately 15-20 photoreceptors were present in each sample; only cones with all mitochondria fully visible within the blocks were chosen for reconstruction. Cell membranes were also reconstructed to provide the containing envelope for mitochondria in FDTD simulations. Counts of mitochondria and their volume 
measurements were obtained using the IMODINFO command-line utility included with IMOD and verified with the Print3D plugin for Blender v2.9 (https://www.blender.org).

To obtain mitochondrial skeletons for alignment analysis, reconstructed models were first loaded into Blender, oriented to point in the $+Z$ direction, and rendered as a series of high-contrast images (i.e., a synthetic Z-stack) for further processing. These processed image stacks were then converted into a format readable by KNOSSOS v5.1그 (https://www.knossos.app), which was then used to load and annotate each mitochondrion as an individual skeleton. One final skeleton was then annotated using the approximate center point of the cone to define the "primary axis" of each cone cell as a function of Z. This two-step approach was adopted instead of directly annotating the SBEM image datasets because of the difficulty of identifying mitochondrial centers in the slicing plane, which was typically oblique to the primary cone axis (see Fig. 2a). Furthermore, renders of reconstructions incorporated the existing "interpretation" of structures that had already been generated from manual contour tracing, thus improving accuracy.

Orientation and alignment analysis were performed on mitochondria skeletons as follows. Skeleton XML data files generated by KNOSSOS were imported using Matlab R2016b (Mathworks, Natick MA). Each mitochondrial skeleton was defined by several nodes connected by links (mitochondrial branches). From the bottom (-Z direction) to the top ( $+Z)$ of the cell volume, the $Z$ axis was discretized into $0.5 \mathrm{~mm}$ steps, and at each of these $Z$ steps (i.e., each height layer), we computed the angle between the primary cone branch at that height (see the previous paragraph for the definition of the primary cone skeleton) and each mitochondrial branch passing through that height layer (see Fig. 2D for an illustration of this calculation). For a single mitochondrion passing through this height layer multiple times, each branch was considered separately. Suppl. Fig. 3 depicts the normalized histograms of mitochondria angles as a function of $Z$ (height) for each reconstructed cell; the graph in Fig. 2D shows the average histogram across all height levels for mitochondria reconstructions from active vs. hibernating GS cones.

\section{D stack light concentration analysis}

Light concentration by cones was measured in Z-stacks using custom routes written in Matlab R2016b. Because of the similar structure of data between confocal imaging and FDTD electromagnetic simulations (see below), this analysis was applied to both experimental and simulated light transmission data.

Briefly, ellipsoid locations in 3D were annotated by drawing rings around a cone's TMRE fluorescence in successive images in each stack to delineate the approximate bounds of the MtB. This annotation provided both an estimate of the distal IS tip of the cone as well as its orientation angle (calculated from an average axis created by the ring centers, weighted by ring area; see Suppl. Fig. 4D). For each such annotated cone, its concentration of light in the transmitted light channel was then annotated by marking both the point of its peak intensity and a center point close to the center of the ellipsoid where the transmitted light forms a "halo" (see Fig. 1D). The line between these two points formed the optical axis along which the light intensity was measured; for each successive image in the stack, the intensity within concentric circles centered upon this axis was calculated. For depth profiles of light 
intensity such as in Fig. 1D (bottom), the intensity within $0.75 \mathrm{~mm}$ of this axis was averaged. To determine the background (unfocused) light level, needed for a calculation of light collection power, an "empty cone" was annotated in each image in a location devoid of structures and featuring a uniform light intensity. The intensity of light collected by annotated cones was then divided by this background intensity to provide a measure of light collection as a function of depth (Z) relative to the distal IS tip.

To provide a measure of the degree to which light available to the apparent OS region depends upon cone ellipsoid angle (i.e., Stiles-Crawford like direction sensitivity), a secondary anatomical axis was defined by the MtB ring annotation as described above (see Fig. 4D). The calculation of light intensity in concentric circles centered upon this anatomical axis was repeated as described for the primary optical axis. These two axes allowed a measure of the rotation angle of the cone ellipsoid compared to the optical axis as well as the calculation of the relative light intensity in the anatomical OSR vs. the optical OSR (i.e., the light available to the OS for a cone ellipsoid perfectly aligned to the optical axis; see Fig. 4D). To facilitate comparison to human direction sensitivity parameters, a pupil-displacement to incident-angle conversion of $2.5 \% \mathrm{~mm}$ was assumed, corresponding approximately to a $25 \mathrm{~mm}$ eye diameter. For simulations of tilted cone reconstructions, angles were measured from the resulting data using this analysis of anatomical vs. optical axes instead of assuming the rotation angles used when building and rotating model structures prior to simulation (see below).

\section{FDTD electromagnetic simulations}

The simulation of light transmission through reconstructed cones and their mitochondria was accomplished via the calculation of Maxwell's equations of electromagnetism (EM) in a discretized grid containing the reconstructed models, into which EM energy oscillating at visible wavelengths was introduced. This process was comprised of four fundamental steps: mesh conditioning, model conversion, EM calculations (simulation), and data analysis. Conversion and simulation procedures were performed using custom $\mathrm{C}++$ programs and executed on Biowulf, the NIH supercluster.

\section{Pre-conversion mesh conditioning}

Manual segmentation of cone mitochondria produced 3D meshes defining the membrane boundaries of mitochondria and the cell inner segment. Prior to conversion, models were rotated to orient the inner segment orthogonally in the $+Z$ direction; for simulations considering the angular dependence of light detection, models were then additionally rotated in the $\mathrm{Y}$-axis by fixed angles. Mitochondria and cell membrane models containing artifacts that resulted from imaging jitter or segmentation errors were edited in Blender software to remove spurious sharp edges or gaps in meshes. Meshes were further decimated to reduce the number of triangles used in model conversion (see below). In models where the inner segment membrane was cut off by the edge of the SBEM imaging volume, the cell membrane mesh was edited in Blender to provide a reasonable approximation to the actual expected morphology for simulations. This workflow of converting 3D meshes to finite difference time-domain simulation (FDTD)-compatible structures further allowed the construction of de novo alternative mitochondria models for simulation (see Suppl. Fig. 6).

\section{Conversion}


To translate these oriented meshes into a 3D gridded structure for FDTD simulations (see below), each grid point was tested against the mesh using a point-in-polygon algorithm $\underline{68}$ and the resulting grid stored as a file for later use (Suppl. Fig. 4B). This storage method allowed for the assignment of varying dielectric parameters (refractive indices) to different structures during simulation without the need to re-compute the structure. Because the Yee grid formulation used in FDTD simulations computes electric and magnetic fields offset by one-half grid spacing from one another $\underline{35}$, this grid discretization was performed at double the resolution specified for actual simulations (i.e., $0.01 \mathrm{~mm}$ grid spacing for simulations using a $0.02 \mathrm{~mm}$ grid). To speed computation, each mitochondrial mesh was discretized into "slabs" in Z containing mesh triangles that intersected a given slab; thus, each point-in-polygon check was simplified to a 2D calculation involving only local triangles. To improve FDTD simulation accuracy using subpixel averaging $\underline{69}$, grid points intersecting cell membranes were detected and stored separately with the normal vector of the triangle intersecting that point as well as the volumetric fill fraction calculated for that point (e.g., for a mitochondrial membrane, the proportion of a given grid point considered "inside" the mitochondrion). These values were later loaded into simulations to initialize the anisotropic dielectric tensor at that point $\underline{69}$. Cell and mitochondrial membranes were assumed to have a width of $10 \mathrm{~nm}$; subpixel averaging allowed the elevated refractive indices of membranes to affect light propagation despite their sub-20 $\mathrm{nm}$ width.

\section{FDTD Electromagnetic Simulation}

Models converted as described above were then pre-loaded with custom $\mathrm{C}++$ code that used libraries from MEEP 1.2.1 ${ }^{35}$ as the foundation for FDTD simulations. For simulations, the converted 3D grids were centered in the $X-Y$ plane with the IS tip placed at a fixed $Z$ height of $25 \mathrm{~mm}$; the simulation volume was further extended $10 \mathrm{~mm}$ beyond the IS tip to allow space for the calculated EM fields to evolve (Fig. 3, Suppl. Fig. 4C). If the total converted model grid length was shorter than $25 \mathrm{~mm}$ in length, the first layer of the converted model grid was repeated in the $-Z$ direction to provide a continuous homogeneous structure. On all sides, a $1.0 \mathrm{~mm}$-thick perfectly matched layer (PML) was established to absorb energy leaving the simulation volume and avoid spurious interference from boundary reflections. Light was launched as a continuous plane wave (Ex-polarized current source with a wavelength in the visible range, typically $450 \mathrm{~nm}$ ) filling the $X-Y$ plane and at a $Z$ height of either 9 $\mathrm{mm}$ ("close source") or $5 \mathrm{~mm}$ ("far source;" see Suppl. Fig. 7). Sources were launched from Z = $9 \mathrm{~mm}$ unless specified otherwise. The grid was divided into 50 steps per unit length, with models scaled to each length unit equaling $1.0 \mathrm{~mm}$; this corresponds to a grid resolution of $20 \mathrm{~nm}$. To allow EM waves to propagate through the simulation volume, fields were iteratively calculated until 50.0 simulation unit time steps; with a length unit of $1 \mathrm{~mm}$, this corresponds to $50 \times 1 \mathrm{e}-6 \mathrm{~m} / c_{0}=1.6678 \mathrm{e}-13 \mathrm{~s}$ or 166.78 femtosec (where $c_{0}$ is the speed of light in a vacuum: $299792458.0 \mathrm{~m} / \mathrm{s}$ ).

For these simulations, the dielectric model grid was specified using the relative permittivity $\varepsilon_{r}=n^{2}$, where $n$ is the refractive index of each material type (e.g., membrane or cytoplasm) in the simulation. Absorption (conductivity of the material) was not modeled. Refractive indices, which may be wavelength dependent, were not modified specifically for the source wavelengths used here. The values for refractive indices were based on previous reports of these values for biological tissues in general $\underline{20,21,34,70-72}$ as well as for photoreceptors in particular $\underline{48}$. Here, unless otherwise specified, the refractive indices used were: membrane, 1.46; cytoplasm, 1.36; mitochondria, 1.40; extracellular space, 1.34. In simulations including procedurally generated cristae structures (see below), the 
mitochondrial refractive index was instead divided into separate intramembrane and inner matrix compartments with separate refractive indices (see Suppl. Fig. 5).

For simulations including procedurally generated cristae structures (Suppl. Fig. 5), instead of uniform average refractive indices surrounded by a membrane envelope, mitochondria were filled by randomized compartments representing the mitochondrial inner matrix and intramembrane space, separated by a second internal membrane. These cristae were constructed using a sum of 3D cosine functions $(f)$ with randomly assigned spatial frequencies and phases, whose values were chosen to qualitatively approximate the spacing of structures present in SBEM images. Grid locations where this sum was positive were assigned as inner matrix; negative values were assigned as intramembrane space. Locations where the absolute value of this sum divided by the magnitude of its gradient was less than half the membrane thickness were assigned as internal membrane (i.e., $|f /\|\nabla f\||<5 \mathrm{~nm}$ ). Following these assignments, dielectric properties were initialized as described elsewhere in this study. The choices of spatial frequencies were isotropic; i.e., this simplification made no assumption regarding any particular orientation or pattern of cristae structures beyond the intent to create refractive index variations spatially modulated using frequencies on the order of those observed.

\section{Analysis}

At the end of each simulation, the dielectric $\varepsilon_{r}$ value (see above) and the EM energy density (EnergyDensity) were exported over the entire simulation volume. These files were then converted into image Z-stacks using custom Matlab scripts; these images were loaded and analyzed as described above for Z-stacks acquired with confocal imaging, where the $\varepsilon_{r}$ stack served as a structural marker analogous to the TMRE channel, and the energy density was measured as for the transmitted ("blue") channel above. To obtain a measurement of the "background" unfocused light energy, brief simulations were performed with no cone structure ("empty" simulations) using a constant refractive index throughout the simulation volume that was equal to that of the extracellular space.

\section{$\underline{\text { Statistics }}$}

For comparisons between active and hibernating GS involving reconstructions or simulations of light transmission through reconstructed models, statistical analysis was not performed due to the low effective sample size of $n=1$ (one retina each from active vs. hibernating animals). For comparisons of simulated light gathering by reconstructed cones with varying configurations (e.g., normal vs. empty IS), Two-sample, two-tailed t-tests were performed in Matlab, with significance markings as follows: *: $p<0.05 ;^{* *}: p<0.01 ; * * * p<0.001$. Throughout the present study, focal lengths and peak concentration factors are reported, but statistical comparisons are only presented for OSR concentration, both because the prior two measurements are limited by the distal extent of the data volume (especially so in simulations, see e.g., Fig. $3 \mathrm{C}$ ) and because the concentration of light in the OSR is the more physiologically relevant measure for light detection by photoreceptors.

For experimental comparisons of light concentration by cones from horizontally sliced GS retina, the mean difference of light concentration in the apparent OS region between samples from active and hibernating GS was determined by bootstrap statistical sampling with replacement (see Suppl. Fig. 8). Samples were structured to match the original experiment, i.e., 4 animals each in either the awake or hibernating condition, with each animal producing a pool of cones for analysis. The pool sizes for 
bioRxiv preprint doi: https://doi.org/10.1101/2021.02.11.430818; this version posted April 8, 2021. The copyright holder for this preprint (which was not certified by peer review) is the author/funder, who has granted bioRxiv a license to display the preprint in perpetuity. It is made available under aCC-BY 4.0 International license.

bootstrap resampling were chosen to match those achieved in the real experiments. Such sampling was repeated $10 \mathrm{k}$ times and the differences between the means of active vs. hibernating samples were recorded. The range of differences reported in Fig. 2E corresponds to the 0.5 and 99.5 percentile values over the population of resampled mean differences (i.e., the $99 \%$ confidence interval). 


\section{References}

1. Brücke, E. About the physiological meaning of the rod-shaped bodies and the twin cones in the eyes of the vertebrates. Archiv für Anatomie, Physiologie und Wissenschaftliche Medicin 11, (1844).

2. Roorda, A. \& Williams, D. R. Optical fiber properties of individual human cones. Journal of Vision 2, 4-4 (2002).

3. Scoles, D. et al. In Vivo Imaging of Human Cone Photoreceptor Inner Segments. Invest. Ophthalmol. Vis. Sci. 55, 4244-4251 (2014).

4. Solovei, I. et al. Nuclear Architecture of Rod Photoreceptor Cells Adapts to Vision in Mammalian Evolution. Cell 137, 356-368 (2009).

5. Franze, K. et al. Müller cells are living optical fibers in the vertebrate retina. PNAS 104, 8287-8292 (2007).

6. Agte, S. et al. Müller Glial Cell-Provided Cellular Light Guidance through the Vital Guinea-Pig Retina. Biophysical Journal 101, 2611-2619 (2011).

7. Labin, A. M., Safuri, S. K., Ribak, E. N. \& Perlman, I. Müller cells separate between wavelengths to improve day vision with minimal effect upon night vision. Nat Commun 5, 1-9 (2014).

8. Westheimer, G. Directional sensitivity of the retina: 75 years of Stiles-Crawford effect. Proc Biol Sci 275, 2777-2786 (2008).

9. Snyder, A. W. \& Pask, C. The Stiles-Crawford effect-explanation and consequences. Vision Research 13, 1115-1137 (1973).

10. Toomey, M. B. \& Corbo, J. C. Evolution, Development and Function of Vertebrate Cone Oil Droplets. Front. Neural Circuits 11, (2017).

11. Young, S. R. \& Martin, G. R. Optics of retinal oil droplets:A model of light collection and polarization detection in the avian retina. Vision Research 24, 129-137 (1984). 
12. Ives, J. T., Normann, R. A. \& Barber, P. W. Light intensification by cone oil droplets: electromagnetic considerations. J. Opt. Soc. Am., JOSA 73, 1725-1731 (1983).

13. Baylor, D. A. \& Fettiplace, R. Light path and photon capture in turtle photoreceptors. The Journal of Physiology 248, 433-464 (1975).

14. Stavenga, D. G. \& Wilts, B. D. Oil droplets of bird eyes: microlenses acting as spectral filters. Philos Trans R Soc Lond B Biol Sci 369, 20130041 (2014).

15. Wilby, D. et al. Optics of cone photoreceptors in the chicken (Gallus gallus domesticus). Journal of The Royal Society Interface 12, 20150591 (2015).

16. Wilby, D. \& Roberts, N. W. Optical influence of oil droplets on cone photoreceptor sensitivity. The Journal of Experimental Biology 220, 1997 (2017).

17. Hoang, Q. V., Linsenmeier, R. A., Chung, C. K. \& Curcio, C. A. Photoreceptor inner segments in monkey and human retina: Mitochondrial density, optics, and regional variation. Visual Neuroscience 19, 395-407 (2002).

18. Spaide, R. F. \& Curcio, C. A. Anatomical correlates to the bands seen in the outer retina by optical coherence tomography: literature review and model. RETINA 31, 1609-1619 (2011).

19. Frazier, A. E., Kiu, C., Stojanovski, D., Hoogenraad, N. J. \& Ryan, M. T. Mitochondrial morphology and distribution in mammalian cells. Biological Chemistry 387, 1551-1558 (2006).

20. Beuthan, J., Minet, O., Helfmann, J., Herrig, M. \& Müller, G. The spatial variation of the refractive index in biological cells. Phys. Med. Biol. 41, 369-382 (1996).

21. Barer, R. \& Joseph, S. Refractometry of Living Cells: Part I. Basic Principles. Journal of Cell Science s3-95, 399-423 (1954).

22. Knabe, W., Skatchkov, S. \& Kuhn, H.-J. "Lens Mitochondria” in the Retinal Cones of the Tree-shrew Tupaia belangeri. Vision Research 37, 267-271 (1997). 
23. Almsherqi, Z., Margadant, F. \& Deng, Y. A look through 'lens' cubic mitochondria. Interface Focus 2, 539-545 (2012).

24. Bringmann, A. et al. The primate fovea: Structure, function and development. Progress in Retinal and Eye Research 66, 49-84 (2018).

25. Luan, Y. et al. Integrated transcriptomic and metabolomic analysis reveals adaptive changes of hibernating retinas. Journal of Cellular Physiology 233, 1434-1445 (2018).

26. Ou, J. et al. iPSCs from a Hibernator Provide a Platform for Studying Cold Adaptation and Its Potential Medical Applications. Cell 173, 851-863.e16 (2018).

27. Ou, J., Rosa, S., Berchowitz, L. E. \& Li, W. Induced pluripotent stem cells as a tool for comparative physiology: lessons from the thirteen-lined ground squirrel. Journal of Experimental Biology 222, (2019).

28. Mehta, N. et al. Presumed foveal bacillary layer detachment in a patient with toxoplasmosis chorioretinitis and pachychoroid disease. Retinal Cases and Brief Reports Publish Ahead of Print, (2021).

29. Kuwabara, T. Cytologic changes of the retina and pigment epithelium during hibernation. Invest Ophthalmol 14, 457-467 (1975).

30. Remé, C. E. \& Young, R. W. The effects of hibernation on cone visual cells in the ground squirrel. Invest. Ophthalmol. Vis. Sci. 16, 815-840 (1977).

31. Sajdak, B. S. et al. Evaluating seasonal changes of cone photoreceptor structure in the 13-lined ground squirrel. Vision Research 158, 90-99 (2019).

32. Frerichs, K. U. et al. Suppression of protein synthesis in brain during hibernation involves inhibition of protein initiation and elongation. PNAS 95, 14511-14516 (1998). 
33. D’Alessandro, A., Nemkov, T., Bogren, L. K., Martin, S. L. \& Hansen, K. C. Comfortably Numb and Back: Plasma Metabolomics Reveals Biochemical Adaptations in the Hibernating 13-Lined Ground Squirrel. J. Proteome Res. 16, 958-969 (2017).

34. Thar, R. \& Kühl, M. Propagation of electromagnetic radiation in mitochondria? Journal of Theoretical Biology 230, 261-270 (2004).

35. Oskooi, A. F. et al. Meep: A flexible free-software package for electromagnetic simulations by the FDTD method. Computer Physics Communications 181, 687-702 (2010).

36. Zhao, H., Brown, P. H. \& Schuck, P. On the Distribution of Protein Refractive Index Increments. Biophysical Journal 100, 2309-2317 (2011).

37. Lluch, S., López-Fuster, M. J. \& Ventura, J. Giant mitochondria in the retina cone inner segments of shrews of genus Sorex (Insectivora, Soricidae). The Anatomical Record Part A: Discoveries in Molecular, Cellular, and Evolutionary Biology 272A, 484-490 (2003).

38. Tarboush, R., Flamarique, I. N., Chapman, G. B. \& Connaughton, V. P. Variability in mitochondria of zebrafish photoreceptor ellipsoids. Visual Neuroscience 31, 11-23 (2014).

39. Yoshimatsu, T., Schröder, C., Nevala, N. E., Berens, P. \& Baden, T. Fovea-like Photoreceptor Specializations Underlie Single UV Cone Driven Prey-Capture Behavior in Zebrafish. Neuron (2020) doi:10.1016/j.neuron.2020.04.021.

40. Nag, T. C. \& Bhattacharjee, J. Retinal ellipsosomes: morphology, development, identification, and comparison with oil droplets. Cell Tissue Res 279, 633-637 (1995).

41. Fisher, S. K. et al. 1 - Light and Electron Microscopy of Vertebrate Photoreceptors: Including a Technique for Electron Microscopic Autoradiography. in Methods in Neurosciences (ed. Hargrave, P. A.) vol. 15 3-36 (Academic Press, 1993). 
42. Hendrickson, A. Organization of the Adult Primate Fovea. in Macular Degeneration (eds. Penfold, P. L. \& Provis, J. M.) 1-23 (Springer, 2005). doi:10.1007/3-540-26977-0_1.

43. Laties, A. M. \& Enoch, J. M. An Analysis of Retinal Receptor Orientation : I. Angular Relationship of Neighboring Photoreceptors. Investigative Ophthalmology \& Visual Science 10, 69-77 (1971).

44. Chai, Z. et al. Light-dependent photoreceptor orientation in mouse retina. Science Advances 6, eabe2782 (2020).

45. Stiles, W. S., Crawford, B. H. \& Parsons, J. H. The luminous efficiency of rays entering the eye pupil at different points. Proceedings of the Royal Society of London. Series B, Containing Papers of a Biological Character 112, 428-450 (1933).

46. Enoch, J. M. Optical Properties of the Retinal Receptors. J. Opt. Soc. Am. 53, 71-85 (1963).

47. Rowe, M. P., Engheta, N., Easter, S. S. \& Pugh, E. N. Graded-index model of a fish double cone exhibits differential polarization sensitivity. J. Opt. Soc. Am. A, JOSAA 11, 55-70 (1994).

48. Sidman, R. L. The structure and concentration of solids in photoreceptor cells studied by refractometry and interference microscopy. The Journal of Biophysical and Biochemical Cytology 3, 15 (1957).

49. Applegate, R. A. \& Lakshminarayanan, V. Parametric representation of Stiles-Crawford functions: normal variation of peak location and directionality. J. Opt. Soc. Am. A, JOSAA 10, 1611-1623 (1993).

50. Stiles, W. S. \& Parsons, J. H. The luminous efficiency of monochromatic rays entering the eye pupil at different points and a new colour effect. Proceedings of the Royal Society of London. Series B Biological Sciences 123, 90-118 (1937).

51. Birch, D. G., Sandberg, M. A. \& Berson, E. L. The Stiles-Crawford effect in retinitis pigmentosa. Invest. Ophthalmol. Vis. Sci. 22, 157-164 (1982). 
52. Enoch, J. M. \& Hope, G. M. Directional Sensitivity of the Foveal and Parafoveal Retina. Invest.

Ophthalmol. Vis. Sci. 12, 497-503 (1973).

53. Westheimer, G. Dependence of the magnitude of the Stiles-Crawford effect on retinal location.

The Journal of Physiology 192, 309-315 (1967).

54. Dowling, J. E. Foveal Receptors of the Monkey Retina: Fine Structure. Science 147, 57-59 (1965).

55. Yamada, E. Some Structural Features of the Fovea Centralis in the Human Retina. Arch Ophthalmol

82, 151-159 (1969).

56. Burns, S. A., Wu, S., He, J. C. \& Elsner, A. E. Variations in photoreceptor directionality across the central retina. J. Opt. Soc. Am. A, JOSAA 14, 2033-2040 (1997).

57. Delint, P. J., Berendschot, T. T. J. M. \& van Norren, D. Local photoreceptor alignment measured with a scanning laser ophthalmoscope. Vision Research 37, 243-248 (1997).

58. Lamb, T. D. Evolution of phototransduction, vertebrate photoreceptors and retina. Progress in Retinal and Eye Research 36, 52-119 (2013).

59. Jonnal, R. S. et al. The Cellular Origins of the Outer Retinal Bands in Optical Coherence Tomography Images. Invest. Ophthalmol. Vis. Sci. 55, 7904-7918 (2014).

60. Ferrington, D. A., Fisher, C. R. \& Kowluru, R. A. Mitochondrial Defects Drive Degenerative Retinal Diseases. Trends in Molecular Medicine 26, 105-118 (2020).

61. Merriman, D. K., Lahvis, G., Jooss, M., Gesicki, J. A. \& Schill, K. Current practices in a captive breeding colony of 13-lined ground squirrels (Ictidomys tridecemlineatus). Lab Anim (NY) 41, 315325 (2012).

62. Jain, A., Yang, A. H. J. \& Erickson, D. Gel-based optical waveguides with live cell encapsulation and integrated microfluidics. Opt. Lett., OL 37, 1472-1474 (2012). 
63. Lee, T.-M. et al. Optical characterization of contrast agents for optical coherence tomography. in Genetically Engineered and Optical Probes for Biomedical Applications vol. 4967 129-134 (International Society for Optics and Photonics, 2003).

64. Mukherjee, K. et al. Analysis of Brain Mitochondria Using Serial Block-Face Scanning Electron Microscopy. JoVE (Journal of Visualized Experiments) e54214 (2016) doi:10.3791/54214.

65. Fiala, J. C. Reconstruct: a free editor for serial section microscopy. Journal of Microscopy 218, 5261 (2005).

66. Kremer, J. R., Mastronarde, D. N. \& McIntosh, J. R. Computer Visualization of Three-Dimensional Image Data Using IMOD. Journal of Structural Biology 116, 71-76 (1996).

67. Helmstaedter, M., Briggman, K. L. \& Denk, W. High-accuracy neurite reconstruction for highthroughput neuroanatomy. Nature Neuroscience 14, 1081-1088 (2011).

68. Hormann, K. \& Agathos, A. The point in polygon problem for arbitrary polygons. Computational Geometry 20, 131-144 (2001).

69. Farjadpour, A. et al. Improving accuracy by subpixel smoothing in the finite-difference time domain. Opt. Lett., OL 31, 2972-2974 (2006).

70. Barer, R. \& Joseph, S. Refractometry of Living Cells: Part II. The Immersion Medium. Journal of Cell Science s3-96, 1-27 (1955).

71. Barer, R. \& Joseph, S. Refractometry of Living Cells: Part III. Technical and Optical Methods. Journal of Cell Science s3-96, 423-447 (1955).

72. Johnsen, S. \& Widder, E. A. The Physical Basis of Transparency in Biological Tissue: Ultrastructure and the Minimization of Light Scattering. Journal of Theoretical Biology 199, 181-198 (1999). 